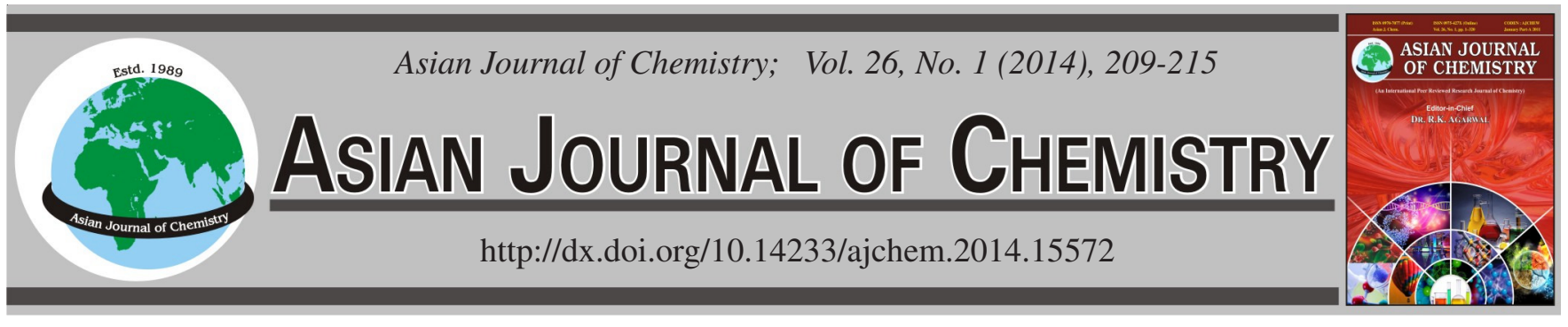

\title{
Design, Synthesis and Antibacterial Evaluation of Novel Fluoroquinolone and its Derivatives
}

Kang-Min Wang ${ }^{1}$, Yuan-Cheng Qin ${ }^{1}$, Guan-Jun Cheng ${ }^{1}$, Hua-Jun Zhu² ${ }^{2}$ Long Liang $^{2}$, Zhi-Peng Cheng ${ }^{2}$ and Mei-Ming Luo ${ }^{1, *}$

${ }^{1}$ Key Laboratory of Green Chemistry and Technology of Ministry of Education at Sichuan University, College of Chemistry, Sichuan University, No. 29 Wangjiang Road, Chengdu 610064, Sichuan Province, P.R. China

${ }^{2}$ Sichuan Kelun Pharmaceutical Co. Ltd, 18, 19/F, 2/Building, Jinjiang Times Garden, 107 Jinli West Road, Chengdu 610067, P.R. China

*Corresponding author: Tel/Fax: +86 28 85462021; E-mail: luomm@ @scu.edu.cn

Received: 3 April 2013;

Accepted: 25 May 2013;

Published online: 26 December 2013;

AJC-14506

Gatifloxacin isomers, [1-cyclopropyl-6-fluoro-1,4-dihydro-8-methoxy-7-(2-methyl-1-piperazinyl)-4-oxo-3-quinoline carboxylic acid] and a series of its derivatives were designed and synthesized and evaluated for their in vitro antibacterial activities. Preliminary results indicated that the tested compounds GI1, GI2, GI3 and GI4 demonstrated excellent activity against Staph. epidermidis. In addition, compounds GI1, GI3 and GI4 show MIC $0.015 \mu \mathrm{g} / \mathrm{mL}$ against Klebsiella peneumoniae. It is worth noting that compound GI2 has been found to exhibit the most prominent activity against all of the tested stains. On the basis of these promising results, some tested compounds could be selected as potential drugs candidate for further evaluation.

Keywords: : Gatifloxacin isomers, 8-Methoxyfluoroquinolones, 2-Methylpiperazine, Antibacterial activities.

\section{INTRODUCTION}

Since the discovery of norfloxacin in the early $1980 \mathrm{~s}^{1}$, fluoroquinolones (FQs) were found to have enhanced pharmacokinetics properties and potent bactericidal activity, when compared to the previous existing bactericidal drugs. However, the extensive clinical use of fluoroquinolones resulted in an increased quinolone resistance among many pathogens ${ }^{2 \mathrm{a}-\mathrm{f}}$. In addition, certain adverse events (e.g. CNS, phototoxicity and arthropathy) became apparent, although the more serious events are rare ${ }^{3 a-b}$. Consequently, great efforts have been devoted towards the synthesis of novel fluoroquinolones in the recent years.

The search for new fluoroquinolones to overcome growing bacterial resistance focuses largely on two approaches. One is to identify novel targets where no pre-existing resistance exists ${ }^{4}$. This approach, although sound in concept, has been met with little success in identifying quality lead matter due to issues associated with target validation and low hit rates from high throughput screening. The second approach is to explore existing clinically proven targets for new chemical matter or modify existing matter with limited or no cross-resistance to existing agents, eliminating the risk of target validation. This approach has been used successfully by a number of researchers to identify new series of fluoroquinolones.

The bactericidal activity of fluoroquinolones is caused by the inhibition of bacterial type-2 topoisomerase (DNA gyrase and topoisomerase IV), which is greatly influenced by the nature of the C-7substituent ${ }^{5-\mathrm{b}}$. According to structure activity relationship (SAR) analysis of fluoroquinolones, modification of the substituents at the $\mathrm{C}-7$ position is the most adaptable site for chemical change and an area that could affect their potency, spectrum and safety. At the C-7, the piperazinyl groups have been utilized with optimal results. Extensively investigated substituents are 3-substituted piperazin-1-yl and 4-substituted derivatives. For example, gatifloxacin is 3-methyl piperazin-1-yl derivative. Pefloxacin, the 4-methylnorfloxacin and other 4-substituted piperazin-1yl prodrugs of norfloxacin were prepared to improve the bioavailability of the parent ${ }^{6 a-c}$. Furthermore, some studies have demonstrated that increasing molecular mass and bulkiness of a substituent at the C-7 position are not barriers to penetration and simply increasing the lipophilic character at C-7 increased the potent activity ${ }^{7}$. The antiproliferative activity of bis-quinolinium choline kinase inhibitors against the HT-29 colon cancer cell line has been improved with a higher lipophilicity of the substituents ${ }^{8}$. It was reported that $8-\mathrm{OCH}_{3}$ fluoroquinolones with $\mathrm{N}^{1}$-cyclopropyl substitution are much more bactericidal activity than C-8 hydrogen analogs ${ }^{9}$. Gatifloxacin ( $4^{\text {th }}$-generation, Fig. 1$)$, having a particularly strong in vitro activity and broad antimicrobial spectrum, could be promising agents ${ }^{10 a, b}$. However, dysglycemia has been noted as the life-threatening adverse effect of gatifloxacin, which led to its withdrawal from the market in the United States in $2006^{11}$. Despite many advances in gatifloxacin, there exsist 
continuous need for novel quinolones to overcome the limitation of gatifloxacin.<smiles>COc1c2c(cc(F)c1N1CCNC(C)C1)C(=O)C(C(=O)O)=CC2C1CC1</smiles>

Gatifloxacin
Gatifloxacin isomer (Gl-H)<smiles>CC1CNCCN1c1c(F)cc2c3c1OC1CC3C1C=C(C(=O)O)C2=O</smiles>

Fig. 1. Gatifloxacin and gatifloxacin isomer (GI-H)

In an effort to discover new classes of antibacterial agents, it was wisely decided to make structure modifications on gatifloxacin. Sekine and his coworkers suggested that the planar feature between the C-7 piperazinyl ring and the naphthyridine nucleus of enoxacin may affect flouroquinolone to interact with the narrow binding site of the enzyme ${ }^{12,13}$. Bulk group on the 7-position, might restrict the free rotation around the bond axis between the C-7 carbon and the piperazinyl $\mathrm{N}-1$ nitrogen in combination with the fluorine atoms at C-6 and methoxyl at C-8 and accordingly make the dihedral angle change, thus possibly tracking the problem of bacterial resistance and dysglycemic effects. Therefore, we here first reported the synthesis of gatifloxacin isomer (Scheme-I) by introducing methyl group at position 2 on the piperazin-1-yl ring, which was determined by ${ }^{1} \mathrm{H} \mathrm{NMR},{ }^{13} \mathrm{C}$ NMR, mass spectrometry analysis. As expected, it show excellent antibacterial activity, particularly against Klebsiella pneunoniae, when compared to gatifloxacin. Furthermore, acylation and alkylation of piperazine are important structural elements ${ }^{14}$, present in a number of investigational and established drugs ${ }^{15}$. It is reported that sulfonyl fluoroquinolones are a new class of antibacterial fluoroquinolones with especially high in vitro activity against Gram-positive bacteria ${ }^{16}$. These research results intensified our interest, herein a number of new derivatives of fluoroquinolones were synthesized by introduction of diversified substituents, including alkyl, acyl and sulfonyl, to the nitrogen atom of the piperazine ring of the gatifloxacin isomer. Moreover, a SAR study is also explored to facilitate the further development of the fluoro-quinolones. The synthesis of ethyl ester derivative 2 has been reported earlier ${ }^{17}$ and serverd as our key intermediate. We developed a practical and efficient synthesis as a general route to gatifloxacin isomer GI-H (Scheme-I).

2-Methylpiperazine was treated with di-tert-butyl dicarbonate to produce Boc-protected 2-methylpiperazine (3) prior to the substitution reaction to avoid the formation of eonormous quanities of gatifloxacin as byproduct and improve selection and purification of 7-C substituted gatifloxacin isomer.

Since direct nucleophilic aromatic substitution of intermediate $\mathbf{2}$ and Boc-protected 2-methylpiperazine met with no success, a new procedure have been designed, in which boron chelate-type intermediate 4 , prepared according to previously published methods ${ }^{18}$, was treated with compound 3 to overcome the diminished reactivity induced by the 8-methoxy group and the steric hindrance induced by methyl neighbouring electrophilic nitrogen atom. The subsequent cleavage step and Boc deprotection could be combined to one-pot process. Namely, refluxing compound $\mathbf{5}$ in methanol during for $12 \mathrm{~h}$ to be sufficient to perform this cleavage, monitored by thin-layer chromatography, then concentrated hydrochloric acid was added and the Boc protective group of compound $\mathbf{6}$ was removed to afford the novel compound GI-H. The whole process is easy to workup without column chromatography purification that makes it possible to scale up.<smiles>CCOC(=O)c1cn(C2CC2)c2c(OC)c(F)c(F)cc2c1=O</smiles><smiles>COc1c2c(cc(F)c1N1CCN(C(=O)OC(C)(C)C)CC1C)N(C1CC1)C=C(C(=O)O)C(=O)N2c1c(F)cc2c(=O)c(C(=O)O)cn(C3CC3)c2c1O</smiles>

Scheme-I: Reactions and conditions: (a) di-tert-butyl dicarbonate (1 equiv), 2-methylpiperazine ( 1 equiv), $\mathrm{CH}_{2} \mathrm{CI}_{2}, 0{ }^{\circ} \mathrm{C}, 1 \mathrm{~h}, 93 \%$ for 3 ; (b) $\mathrm{BF}_{3}$ - $\mathrm{Et}_{2} \mathrm{O}(13$ equiv), ethyl ester derivative ( 1 equiv), THF, reflux, 2d, $56 \%$ for $\mathbf{4}$; (c) boron chelate-type intermediate (1 equiv), Boc-protected 2-methyl piperazine (2.1 equiv), DMSO, $0{ }^{\circ} \mathrm{C}, 0.5 \mathrm{~h}$, then $40{ }^{\circ} \mathrm{C}, 3 \mathrm{~d}, 23 \%$ for $\mathbf{5}$; (d) $\mathrm{CH}_{3} \mathrm{OH}$, reflux, $12 \mathrm{~h}$, then $0{ }^{\circ} \mathrm{C}, \mathrm{HCI}(\mathrm{aq}), 0.5 \mathrm{~h}, 40 \%$ for GI-H 
A series of novel GI-H derivatives (Fig. 2.) were achieved through the versatile and efficient synthetic route as following.

Preparation of N-alkyl-substituted GI: Compounds GI5, GI-7 and GI1-3 were easily prepared by alkylation of GI$\mathbf{H}$ by nucleophilic substitution with their corresponding halohydrocarbons (Scheme-II). For example, compound GI5 was obained by treatment of GTFXS and triethylamine with benzyl chloride in dichloromethane at room temperature.

Preparation of $\mathrm{N}$-acyl and $\mathrm{N}$-sulfonyl substituted GIS: Reaction of GI-H with a suitable acyl chloride, (Scheme-III) in the presence of triethylamine was performed in dichloromethane solution at room temperature, to afford derivatives GI-8, GI-9, GI-10 and GI-11, respectively. Using a suitable anhydride (Scheme-IV) instead of acyl chloride, the acetyl derivative GI-1 and GI-2 was obtained. GI-H under the same condition was treated with the selected sulfonyl chloride, prepared from the corresponding sulfonic acid and thionyl chloride, to provide derivatives (Scheme-IV) GI-3 and GI-6, respectively.

\section{EXPERIMENTAL}

All non-aqueous reactions were performed under an atmosphere of dry Ar (99.99 \%) using oven-dried glassware and anhydrous solvents. All reagents were obtained from commercial sources and used without further purification. Melting points were determined on an XRC-1 capillary melting point apparatus and are uncorrected. Thin layer chromatography was carried out by using Merck silica gel GF254 plates. Lowresolution mass spectra were recorded on a Finnigan Surveyor MSQ instrument. ${ }^{1} \mathrm{H}$ NMR (400 MHz) and ${ }^{13} \mathrm{C}$ NMR (100 $\mathrm{MHz}$ ) spectra were determined on a Bruker-400 spectrometer in DMSO- $d_{6}$ or $\mathrm{CDCl}_{3}$ using tetramethylsilane (TMS) as an internal standard.

1-Cyclopropyl-6-fluoro-1,4-dihydro-8-methoxy-7-(2methyl-1-piperazinyl)-4-oxo-3-quinolinecarboxylic acid (GI-H).

Step-1 Boc Protection of 2-methylpiperazine: Di-tertbutyl dicarbonate $(16.35 \mathrm{~g}, 0.075 \mathrm{~mol})$ in $\mathrm{CH}_{2} \mathrm{Cl}_{2}(50 \mathrm{~mL})$ was added to a solution of racemic 2-methylpiperazine (7.50 g,<smiles>COc1c(N2CCNCC2C)c(F)cc2c(=O)c(C(=O)O)cn(C3CC3)c12</smiles><smiles>COc1c(N2CCN(C(C)=O)CC2C)c(F)cc2c(=O)c(C(=O)O)cn(C3CC3)c12</smiles><smiles>COc1c(N2CCN(C(=O)OC(C)(C)C)CC2C)c(F)cc2c(=O)c(C(=O)O)cn(C3CC3)c12</smiles><smiles>COc1c(N2CCN(S(C)(=O)=O)CC2C)c(F)cc2c(=O)c(C(=O)O)cn(C3CC3)c12</smiles><smiles>COc1c(N2CCN(Cc3ccccc3)CC2C)c(F)cc2c(=O)c(C(=O)O)cn(C3CC3)c12</smiles><smiles>COc1c(N2CCN(S(=O)(=O)c3ccc(C)cc3)CC2C)c(F)cc2c(=O)c(C(=O)O)cn(C3CC3)c12</smiles><smiles>C=CCN1CCN(c2c(F)cc3c(=O)c(C(=O)O)cn(C4CC4)c3c2OC)C(C)C1</smiles><smiles>COc1c(N2CCN(c3cc(C)ccn3)CC2C)c(F)cc2c(=O)c(C(=O)O)cn(C3CC3)c12</smiles><smiles>COc1c(N2CCN(C(=O)c3ccncc3)CC2C)c(F)cc2c(=O)c(C(=O)O)cn(C3C[C@@H]3C)c12</smiles><smiles>COc1c(N2CCN(C(=O)/C=C/c3ccc(F)cc3)CC2C)c(F)cc2c1N(C1CC1)CC(C(=O)O)C2=O</smiles><smiles>COc1c(N2CCN(C(=O)c3cc4ccccc4o3)CC2C)c(F)cc2c(=O)c(C(=O)O)cn(C3CC3)c12</smiles><smiles>COc1c(N2CCN(C(=O)c3ccc(Br)o3)CC2C)c(F)cc2c(=O)c(C(=O)O)cn(C3CC3)c12</smiles>

GI-10<smiles>COc1c(N2CCN(CC(=O)N3CCOCC3)CC2C)c(F)cc2c(=O)c(C(=O)O)cn(C3CC3)c12</smiles>

Fig. 2. Structures of the novel fluoroquinolones 


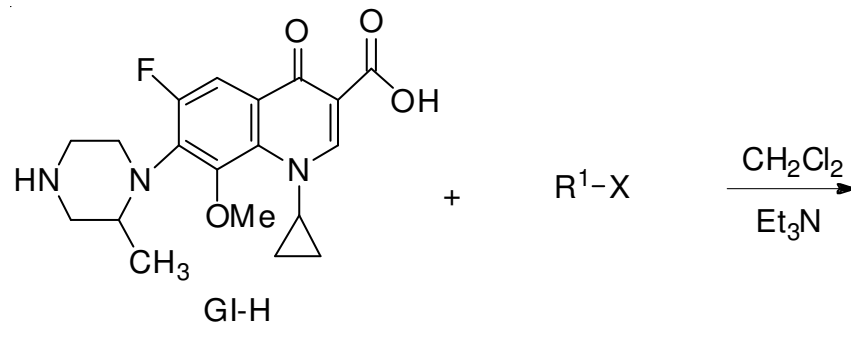

$\mathrm{R}^{1} \mathrm{X}$ :<smiles>ClCc1ccccc1</smiles>

7<smiles>C=CCCl</smiles>

8<smiles>O=C(CCl)N1CCOCC1</smiles>

9<smiles>[R]c1c(N2CCN([R1])CC2C)c(F)cc2c(=O)c(C(=O)O)cn(C3CC3)c12</smiles>

GI-4:<smiles>O=C(Cl)C=CCCl</smiles>

GI-12:<smiles>O=C(CCl)N1CCOCC1</smiles>

Scheme-II: Synthetic route of compound GI-4, GI-6, GI-12

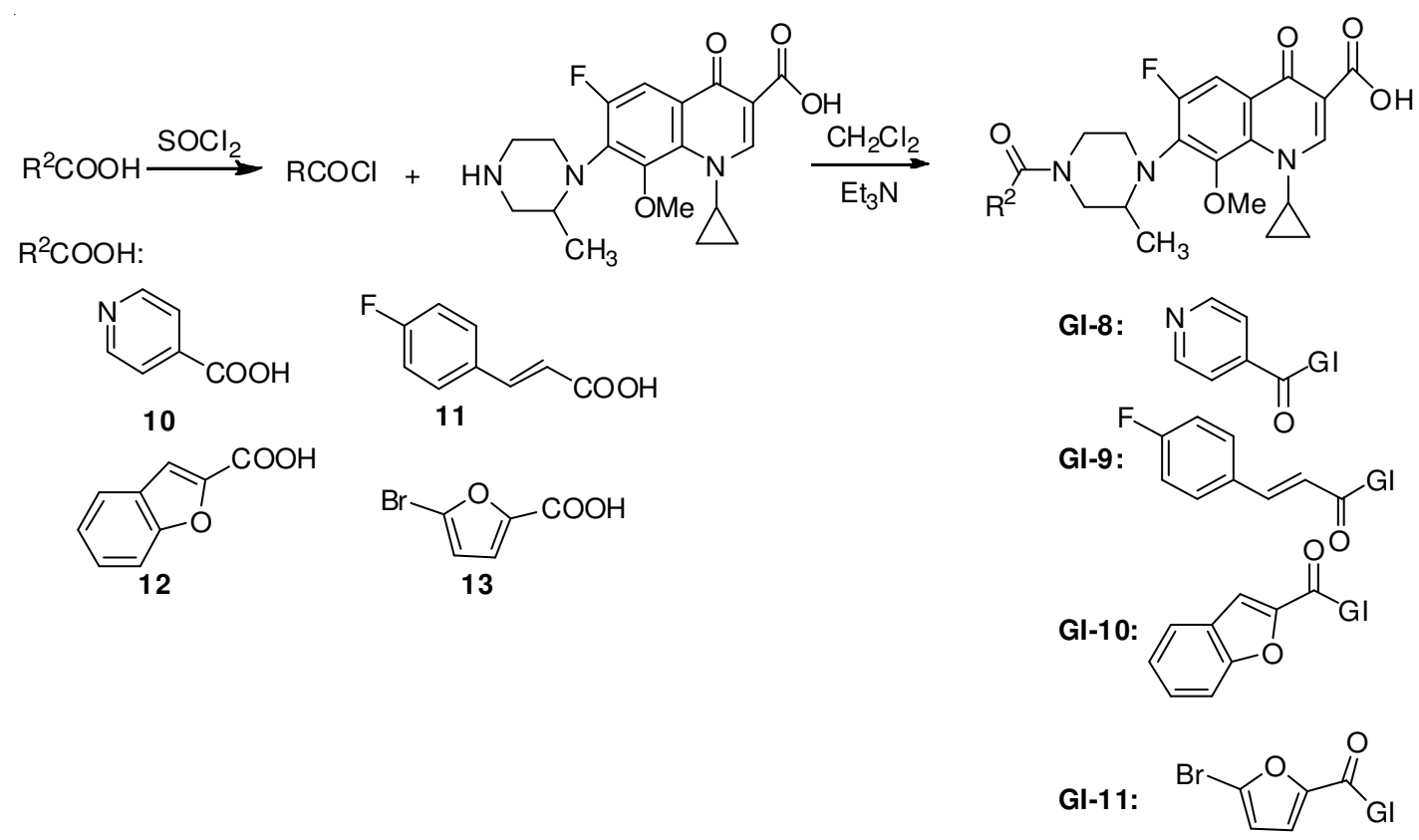

Scheme-III: Synthetic route of compound GI-8, GI-9, GI-10 and GI-11<smiles>COc1c(N2CCNCC2C)c(F)cc2c(=O)c(C(=O)O)cn(C3CC3)c12</smiles>

$R^{3}-\mathrm{C}-\mathrm{O}-\stackrel{\mathrm{O}}{\mathrm{O}}-R^{3} \stackrel{\mathrm{Et}_{3} \mathrm{~N}}{\stackrel{\mathrm{CH}_{2} \mathrm{Cl}_{2}}{\longrightarrow}}$ $\mathbf{R}^{3}=\mathrm{CH}_{3}^{-},\left(\mathrm{CH}_{3}\right)_{3} \mathrm{C}-$.

Gl-1: $\mathrm{CH}_{3} \mathrm{CO}-\mathrm{Gl}$

GI-2: $\left(\mathrm{CH}_{3}\right)_{3} \mathrm{CCO}-\mathrm{GI}$ $+\quad \mathrm{R}^{4}-\mathrm{N}_{\mathrm{N}}^{\mathrm{O}}-\mathrm{Cl} \underset{\mathrm{Et}_{3} \mathrm{~N}}{\stackrel{\mathrm{CH}_{2} \mathrm{Cl}_{2}}{\longrightarrow}}$ $\mathrm{R}^{4}=\mathrm{CH}_{3}$, p-methylphenyl<smiles>[R]C(=O)N1CCN(c2c(F)cc3c(=O)c(C(=O)O)cn(C4CC4)c3c2OC)C(C)C1</smiles> 
$0.075 \mathrm{mmol})$ in $\mathrm{CH}_{2} \mathrm{Cl}_{2}(150 \mathrm{~mL})$ under cooling with salt-ice bath and the whole mixture was stirred at $0{ }^{\circ} \mathrm{C}$ for $1 \mathrm{~h}$. After completion of the reaction, the organic layers was washed with water (three times), dried over sodium sulfate and evaporated in vacuo to give $14 \mathrm{~g}(93 \%)$ of $\mathbf{3}$.

Step-2 Chelation: $\mathrm{BF}_{3} \cdot \mathrm{Et}_{2} \mathrm{O}(36 \mathrm{~mL}, 283.9 \mathrm{mmol})$ was added to a suspension of 2 (7.56 g, $23.4 \mathrm{mmol})$ in THF (150 $\mathrm{mL})$. After refluxing for $48 \mathrm{~h}$, the clear reaction mixture was evaporated under reduced pressure. The crude oily residue was successively washed with $\mathrm{Et}_{2} \mathrm{O}, \mathrm{CHCl}_{3}$ and water affording 4 as a white solid (4.5 g, $56 \%$ ).

Step-3 Coupling: 4 (4.32 g, $12.6 \mathrm{mmol})$ was taken in dimethyl sulfoxide (DMSO), cooled to $0-5{ }^{\circ} \mathrm{C}$. A solution of $\mathbf{3}$ (5.4 g $27 \mathrm{mmol}$ ) in DMSO was slowly added keeping the temperature below $5{ }^{\circ} \mathrm{C}$. The mixture was allowed to stir at $0{ }^{\circ} \mathrm{C}$ for $0.5 \mathrm{~h}$, then warmed to $40^{\circ} \mathrm{C}$ for $3 \mathrm{~d}$. After the reaction ended (TLC monitoring), the mixture was poured into water $(300 \mathrm{~mL})$, forming a generous precipitate. The resulting generous suspension was then cooled at $0-5{ }^{\circ} \mathrm{C}$ and remained at that temperature for an additional hour, then filtered and dried under vacuum to obtain the crude product 5 (1.5 g, $27 \%)$.

Step-4 deprotection: The above crude product 5 was added methanol $(100 \mathrm{~mL})$ and the resulting yellow suspension was heated to reflux $\left(60-65^{\circ} \mathrm{C}\right)$ for $12 \mathrm{~h}$. The mixture was then cooled to $0{ }^{\circ} \mathrm{C}$ and $40 \mathrm{~mL}$ of concentrated aqueous $\mathrm{HCl}$ was carefully added under that temperature. The mixture was stirred for $1 \mathrm{~h}$ at ambient temperature and the solvent was distilled off under reduced pressure until the volume of residue reached $40 \mathrm{~mL}$. The solution was washed with $\mathrm{CH}_{2} \mathrm{Cl}_{2}$ and made alkaline with saturated aqueous $\mathrm{NaOH}$ at $0{ }^{\circ} \mathrm{C}$ and then the $\mathrm{pH}$ of the solution was adjusted to 7.4 with concentrated aqueous $\mathrm{HCl}$ and dilute aqueous $\mathrm{HCl}$. The resultant solution was extracted with chloroform $(4 \times 50 \mathrm{~mL})$ and the combined organic layer was dried over anhydrous $\mathrm{Na}_{2} \mathrm{SO}_{4}$, filtered and concentrated in vacuo. The residue was recrystallized from EtOH to yield crude gatifloxacin isomer GI-H $(0.36 \mathrm{~g}, 24 \%)$ as slightly yellowish white crystals. m.p. $163-164{ }^{\circ} \mathrm{C} . \mathrm{MS}: \mathrm{m} / \mathrm{z}$ calcd. for $\mathrm{C}_{19} \mathrm{H}_{22} \mathrm{~N}_{3} \mathrm{O}_{4} \mathrm{~F}[\mathrm{M}]^{+}: 375.1$; found $[\mathrm{M}+1]^{+}: 376.1 ;{ }^{1} \mathrm{H}$ NMR (400 MHz, $\left.\mathrm{CDCl}_{3}\right): \delta=8.85(\mathrm{~s}, 1 \mathrm{H}), 7.90\left(\mathrm{~d},{ }^{3} J_{\mathrm{F}-\mathrm{H}}=12\right.$ $\mathrm{Hz}, 1 \mathrm{H}), 4.11-4.05(\mathrm{~m}, 1 \mathrm{H}), 3.99$ (s, 3H), 3.66-3.62 (m, 1H), 3.37-3.34 (m, 1H), 3.22-3.16 (m, 2H), 3.11-3.03 (m, 2H), 2.71 $(\mathrm{dd}, J=11.6 \mathrm{~Hz}, 8 \mathrm{~Hz}, 1 \mathrm{H}), 1.32-1.25(\mathrm{~m}, 1 \mathrm{H}), 1.21-1.09(\mathrm{~m}$, $2 \mathrm{H}), 1.05(\mathrm{~d}, J=6.4 \mathrm{~Hz}, 3 \mathrm{H}), 0.95-0.90(\mathrm{~m}, 1 \mathrm{H}) .{ }^{13} \mathrm{C} \mathrm{NMR}$ $\left(100 \mathrm{MHz}, \mathrm{CDCl}_{3}\right): \delta=177.1,166.8,158.8\left(\mathrm{~d},{ }^{1} J_{\mathrm{C}-\mathrm{F}}=252\right.$ $\mathrm{Hz}), 150.1,149.0,138.3\left(\mathrm{~d},{ }^{3} J_{\mathrm{C}-\mathrm{F}}=12 \mathrm{~Hz}\right), 133.4,124.3,124.2$, $107.8\left(\mathrm{~d},{ }^{3} J_{\mathrm{C}-\mathrm{F}}=24 \mathrm{~Hz}\right), 62.5,53.8,53.2,51.5,46.6,40.7$, $16.6,9.9,9.0$.

General procedure for the preparation of compounds GI4, GI-6 and GI-12. To a stirred suspension of gatifluoroxacin isomer ( $1 \mathrm{~g}, 2.5 \mathrm{~mol}), \mathrm{CH}_{2} \mathrm{Cl}_{2}(25 \mathrm{~mL})$ and triethylamine $(0.84$ $\mathrm{mL}, 1.8 \mathrm{~mol}$ ) was added at room temperature. The corresponding halogenated hydrocarbons ( $3 \mathrm{mmol}$ ) and the mixture was stirred at room temperature for $6 \mathrm{~h}$ and then was washed with dilute aqueous $\mathrm{HCl}$ to adjust $\mathrm{pH}$ to 2-3 and finally with water. The organic layer was dried (anhydrous $\mathrm{Na}_{2} \mathrm{SO}_{4}$ ) and evaporated to dryness in vacuo. The solid crystallized from isopropanol to give the derivatives. Compound GI-4: m.p. 120$122{ }^{\circ} \mathrm{C}$. MS: $m / z$ calcd. for $\mathrm{C}_{26} \mathrm{H}_{28} \mathrm{~N}_{3} \mathrm{O}_{4} \mathrm{~F}[\mathrm{M}]^{+}$: 465.2; found $[\mathrm{M}+1]^{+}: 466.2 ;{ }^{1} \mathrm{H} \mathrm{NMR}\left(400 \mathrm{MHz}, \mathrm{CDCl}_{3}\right): \delta=14.71(\mathrm{~s}$,
$1 \mathrm{H}), 8.83(\mathrm{~s}, 1 \mathrm{H}), 7.88\left(\mathrm{~d},{ }^{3} J_{\mathrm{F}-\mathrm{H}}=12 \mathrm{~Hz}, 1 \mathrm{H}\right), 7.36-7.27(\mathrm{~m}$, $5 \mathrm{H}), 4.09-4.03$ (m, 1H), 3.95 (s, 3H), 3.76-3.72 (m, 1H), 3.59$3.54(\mathrm{~m}, 2 \mathrm{H}), 3.42-3.98(\mathrm{~m}, 1 \mathrm{H}), 3.27-3.24(\mathrm{~m}, 1 \mathrm{H}), 2.81-$ $2.68(\mathrm{~m}, 2 \mathrm{H}), 2.53-2.49(\mathrm{~m}, 1 \mathrm{H}), 2.22-2.19(\mathrm{~m}, 1 \mathrm{H}), 1.32-$ $1.28(\mathrm{~m}, 1 \mathrm{H}), 1.21-1.05(\mathrm{~m}, 5 \mathrm{H}), 0.95-0.90(\mathrm{~m}, 1 \mathrm{H}) .{ }^{13} \mathrm{C} \mathrm{NMR}$ $\left(100 \mathrm{MHz}, \mathrm{CDCl}_{3}\right): \delta=177.1,166.7,158.8\left(\mathrm{~d},{ }^{1} J_{\mathrm{C}-\mathrm{F}}=253\right.$ $\mathrm{Hz}), 150.3,148.6,138.4\left(\mathrm{~d},{ }^{3} J_{\mathrm{C}-\mathrm{F}}=13 \mathrm{~Hz}\right), 137.9,133.4,129.1$, 128.3, 127.2, 123.8, 107.7 (d, $\left.{ }^{3} J_{\mathrm{C}-\mathrm{F}}=23 \mathrm{~Hz}\right), 107.6,63.1,62.4$, 60.5, 53.8, 53.4, 53.0, 50.4, 40.7, 29.7, 17.05, 9.9, 8.9.

Compound GI-6: m.p. $158-160{ }^{\circ} \mathrm{C}$. MS: $m / z$ calcd. for $\mathrm{C}_{22} \mathrm{H}_{26} \mathrm{~N}_{3} \mathrm{O}_{4} \mathrm{~F}[\mathrm{M}]^{+}:$415.1; found [M+1] $]^{+}: 416.1 ;{ }^{1} \mathrm{H}$ NMR (400 $\left.\mathrm{MHz}, \mathrm{CDCl}_{3}\right): \delta=14.69(\mathrm{~s}, 1 \mathrm{H}), 8.84(\mathrm{~s}, 1 \mathrm{H}), 7.88\left(\mathrm{~d},{ }^{3} J_{\mathrm{F}-\mathrm{H}}=\right.$ $11.6 \mathrm{~Hz}, 1 \mathrm{H}), 5.94-5.89(\mathrm{~m}, 1 \mathrm{H}), 5.28-5.21$ (m, 2H), 4.10$4.04(\mathrm{~m}, 1 \mathrm{H}), 3.95(\mathrm{~s}, 3 \mathrm{H}), 3.77-3.75(\mathrm{~m}, 1 \mathrm{H}), 3.40-3.29(\mathrm{~m}$, $2 \mathrm{H}), 3.15-3.02(\mathrm{~m}, 2 \mathrm{H}), 2.86-2.79(\mathrm{~m}, 2 \mathrm{H}), 2.55-2.45(\mathrm{~m}, 1 \mathrm{H})$, 2.16-2.02 (m, 1H), 1.28-1.26 (m, 1H), 1.15-1.05 (m, 5H), 0.93$0.83(\mathrm{~m}, 1 \mathrm{H}) .{ }^{13} \mathrm{C} \mathrm{NMR}\left(100 \mathrm{MHz}, \mathrm{CDCl}_{3}\right): \delta=177.0,166.5$, $158.3\left(\mathrm{~d},{ }^{1} J_{\mathrm{C}-\mathrm{F}}=253 \mathrm{~Hz}\right), 150.4,149.0,138.0\left(\mathrm{~d},{ }^{3} J_{\mathrm{C}-\mathrm{F}}=13\right.$ $\mathrm{Hz}), 133.8,133.4,124.1,124.0,119.0,107.6\left(\mathrm{~d},{ }^{3} J_{\mathrm{C}-\mathrm{F}}=18\right.$ Hz), 62.7, 61.6, 60.3, 53.7, 52.6, 50.5, 40.8, 29.6, 17.1, 9.9, 8.9.

Compound GI-12: m.p. $96-98{ }^{\circ} \mathrm{C}$. MS: $\mathrm{m} / \mathrm{z}$ calcd. for $\mathrm{C}_{25} \mathrm{H}_{31} \mathrm{~N}_{4} \mathrm{O}_{6} \mathrm{BrF}[\mathrm{M}]^{+}:$502.2; found $[\mathrm{M}+1]^{+}: 503.2 ;{ }^{1} \mathrm{H} \mathrm{NMR}$ $\left(400 \mathrm{MHz}, \mathrm{CDCl}_{3}\right): \delta=14.66(\mathrm{~s}, 1 \mathrm{H}), 8.84(\mathrm{~s}, 1 \mathrm{H}), 7.90$ (d, $\left.{ }^{3} J_{\mathrm{F}-\mathrm{H}}=11.6 \mathrm{~Hz}, 1 \mathrm{H}\right), 4.10-4.4 .06(\mathrm{~m}, 1 \mathrm{H}), 3.96(\mathrm{~s}, 3 \mathrm{H}), 3.77-$ 3.63 (m, 9H), 3.50-3.40 (m, 1H), 3.27-3.24 (m, 3H), 2.86$2.83(\mathrm{~m}, 1 \mathrm{H}), 2.71-2.65(\mathrm{~m}, 2 \mathrm{H}), 2.34-2.31(\mathrm{~m}, 1 \mathrm{H}), 1.31-$ $1.28(\mathrm{~m}, 1 \mathrm{H}), 1.21-1.11(\mathrm{~m}, 5 \mathrm{H}), 0.95-0.90(\mathrm{~m}, 1 \mathrm{H}) .{ }^{13} \mathrm{C} \mathrm{NMR}$ $\left(150 \mathrm{MHz}, \mathrm{CDCl}_{3}\right): \delta=177.0,167.9,166.6,159.4,150.1$, $148.7,138.1\left(\mathrm{~d},{ }^{3} J_{\mathrm{C}-\mathrm{F}}=12 \mathrm{~Hz}\right), 133.4,124.0,114.3,11.9,107.7$ $\left(\mathrm{d},{ }^{3} J_{\mathrm{C}-\mathrm{F}}=13 \mathrm{~Hz}\right), 67.0,62.4,61.5,60.3,53.8 ., 53.0,49.9$, 46.3, 42.2, 40.7, 16.8, 9.9, 8.9.

General procedure for the preparation of acyl chloride: Acid (10, 11, 12 and 13 ) (12 mmol) was added thionyl chloride $(15 \mathrm{~mL})$ and the mixture was stirred and refluxed for $2 \mathrm{~h}$. Redundant thionyl chloride was distilled off under reduced pressure to afford quantitative yield of the corresponding chloride which was used directly in the next step.

General procedure for the preparation of compounds GI1, GI-2, GI-3, GI-5, GI-7, GI-8, GI-9, GI-10 and GI-11. To a suspension of gatifluoroxacin isomer $(0.7 \mathrm{~g}, 1.8 \mathrm{~mol}), \mathrm{CH}_{2} \mathrm{Cl}_{2}$ $(50 \mathrm{~mL})$ and triethylamine $(0.84 \mathrm{~mL}, 1.8 \mathrm{~mol})$ was dropped in 15 min a solution of fresh acyl chloride prepared by above method or anhydride $(1.8 \mathrm{~mol})$ in $\mathrm{CH}_{2} \mathrm{Cl}_{2}(15 \mathrm{~mL})$. The mixture was stirred at $25^{\circ} \mathrm{C}$ for $0.5 \mathrm{~h}$ and then was washed with dilute aqueous $\mathrm{HCl}$ adjusting $\mathrm{pH}$ to 2-3 and water. The organic layer was dried (anhydrous $\mathrm{Na}_{2} \mathrm{SO}_{4}$ ) and evaporated to dryness in vacuo. The solid crystallized from $80 \% \mathrm{EtOH}$ to afford desired product.

Compound GI-1: m.p. $176-178{ }^{\circ} \mathrm{C}$. MS: $\mathrm{m} / \mathrm{z}$ calcd. for $\mathrm{C}_{21} \mathrm{H}_{24} \mathrm{~N}_{3} \mathrm{O}_{5} \mathrm{~F}[\mathrm{M}]^{+}: 417.1$; found $[\mathrm{M}+1]^{+}:$418.1; ${ }^{1} \mathrm{H}$ NMR (400 $\left.\mathrm{MHz}, \mathrm{CDCl}_{3}\right): \delta=14.60(\mathrm{~s}, 1 \mathrm{H}), 8.83(\mathrm{~s}, 1 \mathrm{H}), 7.90\left(\mathrm{~d},{ }^{3} J_{\mathrm{F}-\mathrm{H}}=\right.$ $11.6 \mathrm{~Hz}, 1 \mathrm{H}), 4.15-3.96(\mathrm{~m}, 2 \mathrm{H}), 3.96(\mathrm{~s}, 3 \mathrm{H}), 3.78-3.62(\mathrm{~m}$, $3 \mathrm{H}), 3.46-3.44$ (m, 2H), 3.32-3.19 (m, 2H), 2.22 (s, 3H), 1.34$1.28(\mathrm{~m}, 1 \mathrm{H}), 1.20-1.08(\mathrm{~m}, 5 \mathrm{H}), 0.96-0.92(\mathrm{~m}, 1 \mathrm{H}) .{ }^{13} \mathrm{C} \mathrm{NMR}$ $\left(100 \mathrm{MHz}, \mathrm{CDCl}_{3}\right): \delta=177.21,166.61,154.83\left(\mathrm{~d},{ }^{1} J_{\mathrm{C}-\mathrm{F}}=261\right.$ $\mathrm{Hz}), 150.17,138.03\left(\mathrm{~d},{ }^{3} J_{\mathrm{C}-\mathrm{F}}=9 \mathrm{~Hz}\right), 133.49,107.96\left(\mathrm{~d},{ }^{3} J_{\mathrm{C}-\mathrm{F}}=\right.$ $23 \mathrm{~Hz}$ ), 80.09,62.5, 53.29, 40.68, 28.44, 16.00, 9.99, 9.04.

Compound GI-2: m.p. $158-160{ }^{\circ} \mathrm{C}$. MS: $\mathrm{m} / \mathrm{z}$ calcd. for $\mathrm{C}_{24} \mathrm{H}_{30} \mathrm{~N}_{3} \mathrm{O}_{6} \mathrm{~F}[\mathrm{M}]^{+}:$475.2; found $[\mathrm{M}+1]^{+}:$476.2; ${ }^{1} \mathrm{H}$ NMR (400 
$\left.\mathrm{MHz}, \mathrm{CDCl}_{3}\right): \delta=14.64(\mathrm{~s}, 1 \mathrm{H}), 8.84(\mathrm{~s}, 1 \mathrm{H}), 7.90\left(\mathrm{~d},{ }^{3} J_{\mathrm{F}-\mathrm{H}}=\right.$ $14.4 \mathrm{~Hz}, 1 \mathrm{H}), 4.10-4.04(\mathrm{~m}, 1 \mathrm{H}), 3.95$ (s, 3H), 3.79-3.73 (m, $1 \mathrm{H}), 3.69-3.66(\mathrm{~m}, 2 \mathrm{H}), 3.55-3.41(\mathrm{~m}, 2 \mathrm{H}), 3.17-3.13(\mathrm{~m}, 2 \mathrm{H})$, $1.50(\mathrm{~s}, 9 \mathrm{H}), 1.36-1.25(\mathrm{~m}, 1 \mathrm{H}), 1.24-1.05(\mathrm{~m}, 5 \mathrm{H}), 0.95-0.85$ $(\mathrm{m}, 2 \mathrm{H}) .{ }^{13} \mathrm{C} \mathrm{NMR}\left(100 \mathrm{MHz}, \mathrm{CDCl}_{3}\right): \delta=177.0,169.3,166.5$, $158.2\left(\mathrm{~d},{ }^{1} J_{\mathrm{C}-\mathrm{F}}=261 \mathrm{~Hz}\right), 150.25,148.51,137.6\left(\mathrm{~d},{ }^{3} J_{\mathrm{C}-\mathrm{F}}=9\right.$ $\mathrm{Hz}), 133.5,124.3,124.2,107.8\left(\mathrm{~d},{ }^{3} J_{\mathrm{C}-\mathrm{F}}=23 \mathrm{~Hz}\right), 62.5,52.8$, 49.8, 49.2, 47.8, 47.1, 42.2, 40.7, 21.3, 21.2, 16.0, 10.0, 9.0.

Compound GI-3: m.p. $178-180{ }^{\circ} \mathrm{C}$. MS: $\mathrm{m} / \mathrm{z}$ calcd. for $\mathrm{C}_{25} \mathrm{H}_{25} \mathrm{~N}_{4} \mathrm{O}_{5} \mathrm{~F}[\mathrm{M}]^{+}:$480.1; found [M+1 $]^{+}: 481.1 ;{ }^{1} \mathrm{H}$ NMR (400 $\left.\mathrm{MHz}, \mathrm{CDCl}_{3}\right): \delta=14.52(\mathrm{~s}, 1 \mathrm{H}), 8.85(\mathrm{~s}, 1 \mathrm{H}), 8.75(\mathrm{~d}, \mathrm{~J}=$ $4 \mathrm{~Hz}, 2 \mathrm{H}), 7.93\left(\mathrm{~d},{ }^{3} J_{\mathrm{F}-\mathrm{H}}=11.6 \mathrm{~Hz}, 1 \mathrm{H}\right), 7.39(\mathrm{~d}, J=4.8 \mathrm{~Hz}$, 2H), 4.29-4.03 (m, 2H), 3.97 (s, 3H), 3.92-3.78 (m, 1H), 3.62$3.53(\mathrm{~m}, 3 \mathrm{H}), 3.40-3.19(\mathrm{~m}, 2 \mathrm{H}), 1.31-1.26(\mathrm{~m}, 1 \mathrm{H}), 1.21-$ $1.11(\mathrm{~m}, 5 \mathrm{H}), 0.95-0.90(\mathrm{~m}, 1 \mathrm{H}) .{ }^{13} \mathrm{C} \mathrm{NMR}\left(100 \mathrm{MHz}, \mathrm{CDCl}_{3}\right)$ : $\delta=177.0,176.9,168.0,166.4,150.3,148.5,143.2,137.4(\mathrm{~d}$, $\left.{ }^{3} J_{\mathrm{C}-\mathrm{F}}=13 \mathrm{~Hz}\right), 133.4,124.4,124.3,107.6\left(\mathrm{~d},{ }^{3} J_{\mathrm{C}-\mathrm{F}}=22 \mathrm{~Hz}\right)$, 62.6, 53.6, 53.7, 50.0, 48.9, 48.3, 42.7, 40.7, 30.9, 16.0, 15.8, 9.9, 9.0.

Compound GI-5: m.p. $174-176{ }^{\circ} \mathrm{C}$. MS: $\mathrm{m} / \mathrm{z}$ calcd. for $\mathrm{C}_{28} \mathrm{H}_{26} \mathrm{~N}_{3} \mathrm{O}_{5} \mathrm{~F}[\mathrm{M}]^{+}:$: 19.1 ; found $[\mathrm{M}+1]^{+}:$520.1; ${ }^{1} \mathrm{H}$ NMR (400 $\left.\mathrm{MHz}, \mathrm{CDCl}_{3}\right): \delta=14.60(\mathrm{~s}, 1 \mathrm{H}), 8.85(\mathrm{~s}, 1 \mathrm{H}), 7.92\left(\mathrm{~d},{ }^{3} J_{\mathrm{F}-\mathrm{H}}=\right.$ $11.6 \mathrm{~Hz}, 1 \mathrm{H}), 7.69$ (d, $J=8 \mathrm{~Hz}, 1 \mathrm{H}), 7.54(\mathrm{~d}, J=8 \mathrm{~Hz}, 1 \mathrm{H})$, 7.44-7.40 (m, 2H), 7.34-7.30 (m, 1H), 4.29-4.03 (m, 2H), 3.98$3.82(\mathrm{~m}, 4 \mathrm{H}), 3.82-3.70(\mathrm{~m}, 1 \mathrm{H}), 3.59-3.56(\mathrm{~m}, 3 \mathrm{H}), 3.32-$ $3.30(\mathrm{~m}, 1 \mathrm{H}), 1.32-1.30(\mathrm{~m}, 1 \mathrm{H}), 1.21-1.11(\mathrm{~m}, 5 \mathrm{H}), 0.95-$ $0.92(\mathrm{~m}, 1 \mathrm{H}) .{ }^{13} \mathrm{C} \mathrm{NMR}\left(100 \mathrm{MHz}, \mathrm{CDCl}_{3}\right): \delta=177.0,176.9$, $166.4,160.0,159.4,156.9,154.6,150.2,148.8,137.7$ (d, ${ }^{3} J_{\mathrm{C}-\mathrm{F}}$ $=14 \mathrm{~Hz}), 126.8,126.6,124.1,124.0,123.7,122.3,122.3$, $112.5,107.7\left(\mathrm{~d},{ }^{3} J_{\mathrm{C}-\mathrm{F}}=20 \mathrm{~Hz}\right), 64.02,62.6,53.6,49.6,40.7$, 25.3, 16.0, 9.9, 9.0.

Compound GI-8: m.p. $178-180{ }^{\circ} \mathrm{C}$. MS: $m / z$ calcd. for $\mathrm{C}_{25} \mathrm{H}_{25} \mathrm{~N}_{4} \mathrm{O}_{5} \mathrm{~F}[\mathrm{M}]^{+}: 480.1$; found $[\mathrm{M}+1]^{+}: 481.1 ;{ }^{1} \mathrm{H}$ NMR (400 $\left.\mathrm{MHz}, \mathrm{CDCl}_{3}\right): \delta=14.52(\mathrm{~s}, 1 \mathrm{H}), 8.85(\mathrm{~s}, 1 \mathrm{H}), 8.75(\mathrm{~d}, J=4$ $\mathrm{Hz}, 2 \mathrm{H}), 7.93\left(\mathrm{~d},{ }^{3} J_{\mathrm{F}-\mathrm{H}}=11.6 \mathrm{~Hz}, 1 \mathrm{H}\right), 7.39(\mathrm{~d}, J=4.8 \mathrm{~Hz}$, $2 \mathrm{H}), 4.29-4.03$ (m, 2H), 3.97 (s, 3H), 3.92-3.78 (m, 1H), 3.623.53 (m, 3H), 3.40-3.19 (m, 2H), 1.31-1.26 (m, 1H), 1.21$1.11(\mathrm{~m}, 5 \mathrm{H}), 0.95-0.90(\mathrm{~m}, 1 \mathrm{H}) .{ }^{13} \mathrm{C} \mathrm{NMR}\left(100 \mathrm{MHz}, \mathrm{CDCl}_{3}\right)$ : $\delta=177.0,176.9,168.0,166.4,150.3,148.5,143.2,137.4(\mathrm{~d}$, $\left.{ }^{3} J_{\mathrm{C}-\mathrm{F}}=13 \mathrm{~Hz}\right), 133.4,124.4,124.3,107.6\left(\mathrm{~d},{ }^{3} J_{\mathrm{C}-\mathrm{F}}=22 \mathrm{~Hz}\right)$, 62.6, 53.6, 53.7, 50.0, 48.9, 48.3, 42.7, 40.7, 30.9, 16.0, 15.8, 9.9, 9.0.

Compound GI-9: m.p. $200-202{ }^{\circ} \mathrm{C}$. MS: $m / z$ calcd. for $\mathrm{C}_{28} \mathrm{H}_{27} \mathrm{~N}_{3} \mathrm{O}_{5} \mathrm{~F}_{2}[\mathrm{M}]^{+}:$523.1; found $[\mathrm{M}+1]^{+}:$524.1; ${ }^{1} \mathrm{H}$ NMR (400 $\left.\mathrm{MHz}, \mathrm{CDCl}_{3}\right): \delta=14.62(\mathrm{~s}, 1 \mathrm{H}), 8.85(\mathrm{~s}, 1 \mathrm{H}), 7.92\left(\mathrm{~d},{ }^{3} J_{\mathrm{F}-\mathrm{H}}=\right.$ $11.6 \mathrm{~Hz}, 1 \mathrm{H}), 7.71(\mathrm{~d}, J=15.2 \mathrm{~Hz}, 2 \mathrm{H}), 7.54(\mathrm{dd}, J=8.4 \mathrm{~Hz}$, $J=5.6 \mathrm{~Hz}, 2 \mathrm{H}), 7.10$ (t, 2H), 6.80 (d, $J=15.2 \mathrm{~Hz}, 1 \mathrm{H}), 4.29$ $4.04(\mathrm{~m}, 2 \mathrm{H}), 3.97-3.82(\mathrm{~m}, 4 \mathrm{H}), 3.82-3.70(\mathrm{~m}, 2 \mathrm{H}), 3.53-$ $3.25(\mathrm{~m}, 3 \mathrm{H}), 1.32-1.26(\mathrm{~m}, 1 \mathrm{H}), 1.21-1.11(\mathrm{~m}, 5 \mathrm{H}), 0.95-$ $0.92(\mathrm{~m}, 1 \mathrm{H}) .{ }^{13} \mathrm{C}$ NMR $\left(100 \mathrm{MHz}, \mathrm{CDCl}_{3}\right): \delta=177.0,176.9$, 166.4, 165.5, 164.7, 162.3, 159.4, 156.9, 150.1, 148.5, 141.9, $137.4\left(\mathrm{~d},{ }^{3} J_{\mathrm{C}-\mathrm{F}}=13 \mathrm{~Hz}\right), 130.9,129.6,124.2,124.1,116.4$, $116.0,107.7\left(\mathrm{~d},{ }^{3} J_{\mathrm{C}-\mathrm{F}}=22 \mathrm{~Hz}\right), 77.3,62.6,48.4,46.6,40.7$, 25.3, 16.1, 10.0, 9.0.

Compound GI-10: m.p. $174-176{ }^{\circ} \mathrm{C}$. MS: $m / z$ calcd. for $\mathrm{C}_{28} \mathrm{H}_{26} \mathrm{~N}_{3} \mathrm{O}_{5} \mathrm{~F}[\mathrm{M}]^{+}:$: 19.1 ; found $[\mathrm{M}+1]^{+}: 520.1 ;{ }^{1} \mathrm{H}$ NMR (400 $\left.\mathrm{MHz}, \mathrm{CDCl}_{3}\right): \delta=14.60(\mathrm{~s}, 1 \mathrm{H}), 8.85(\mathrm{~s}, 1 \mathrm{H}), 7.92\left(\mathrm{~d},{ }^{3} J_{\mathrm{F}-\mathrm{H}}=\right.$ $11.6 \mathrm{~Hz}, 1 \mathrm{H}), 7.69$ (d, $J=8 \mathrm{~Hz}, 1 \mathrm{H}), 7.54(\mathrm{~d}, J=8 \mathrm{~Hz}, 1 \mathrm{H})$,
7.44-7.40 (m, 2H), 7.34-7.30 (m, 1H), 4.29-4.03 (m, 2H), 3.98$3.82(\mathrm{~m}, 4 \mathrm{H}), 3.82-3.70(\mathrm{~m}, 1 \mathrm{H}), 3.59-3.56(\mathrm{~m}, 3 \mathrm{H}), 3.32-$ $3.30(\mathrm{~m}, 1 \mathrm{H}), 1.32-1.30(\mathrm{~m}, 1 \mathrm{H}), 1.21-1.11(\mathrm{~m}, 5 \mathrm{H}), 0.95-$ $0.92(\mathrm{~m}, 1 \mathrm{H}) .{ }^{13} \mathrm{C} \mathrm{NMR}\left(100 \mathrm{MHz}, \mathrm{CDCl}_{3}\right): \delta=177.0,176.9$, $166.4,160.0,159.4,156.9,154.6,150.2,148.8,137.7\left(\mathrm{~d},{ }^{3} J_{\mathrm{C}-\mathrm{F}}\right.$ $=14 \mathrm{~Hz}), 126.8,126.6,124.1,124.0,123.7,122.3,122.3$, $112.5,107.7\left(\mathrm{~d},{ }^{3} J_{\mathrm{C}-\mathrm{F}}=20 \mathrm{~Hz}\right), 64.02,62.6,53.6,49.6,40.7$, 25.3, 16.0, 9.9, 9.0.

Compound GI-11: m.p. $176-178^{\circ} \mathrm{C}$. MS: $m / z$ calcd. for $\mathrm{C}_{24} \mathrm{H}_{23} \mathrm{~N}_{3} \mathrm{O}_{6} \mathrm{BrF}[\mathrm{M}]^{+}: 547.1$; found $[\mathrm{M}+1]^{+}: 548.1 ;{ }^{1} \mathrm{H} \mathrm{NMR}$ $\left(400 \mathrm{MHz}, \mathrm{CDCl}_{3}\right): \delta=14.58(\mathrm{~s}, 1 \mathrm{H}), 8.85(\mathrm{~s}, 1 \mathrm{H}), 7.92(\mathrm{~d}$, $\left.{ }^{3} J_{\mathrm{F}-\mathrm{H}}=11.6 \mathrm{~Hz}, 1 \mathrm{H}\right), 7.07(\mathrm{~d}, J=3.6 \mathrm{~Hz}, 1 \mathrm{H}), 6.47(\mathrm{~d}, J=3.2$ $\mathrm{Hz}, 1 \mathrm{H}), 4.21-4.17$ (m, 1H), 4.10-4.4.04 (m, 2H), 3.96 (s, 3H), 3.88-3.77 (m, 2H), 3.57-3.53 (m, 2H), 3.32-3.24 (m, 1H), 1.32$1.30(\mathrm{~m}, 1 \mathrm{H}), 1.21-1.11(\mathrm{~m}, 5 \mathrm{H}), 0.95-0.90(\mathrm{~m}, 1 \mathrm{H}) .{ }^{13} \mathrm{C} \mathrm{NMR}$ $\left(100 \mathrm{MHz}, \mathrm{CDCl}_{3}\right): \delta=177.0,176.9,166.4,159.4,158.0$, $156.9,150.2,149.4,148.5,137.6\left(\mathrm{~d},{ }^{3} J_{\mathrm{C}-\mathrm{F}}=13 \mathrm{~Hz}\right), 133.5$, $124.3,124.0,119.3,113.5,107.8\left(\mathrm{~d},{ }^{3} J_{\mathrm{C}-\mathrm{F}}=20 \mathrm{~Hz}\right), 77.3,62.5$, $53.5,49.5,40.7,16.0,10.0,9.0$.

\section{RESULTS AND DISCUSSION}

Each compound was screened for in vitro antibacterial activity against several representative Gram-positive bacteria (S. aureus, S. aureus and S. epidermidis) and Gram-negative bacteria (Escherichia coli, P. aeuginosa, K. peneumoniae, Acinetobacter baumannii). The minimum inhibitory concentration values (MICs) were determined by comparison to its isomer, gatifloxacin as reference drug (Table-1) ${ }^{19}$.

Generally, all of the synthesized compounds exhibited potent antibacterial activity. Most tested compounds possess good activity against $E$. coli and $S$. epidermidis, particularly GI-1 and GI-II. The most prominent improvement was observed against Klebsiella peneumoniae. The compounds GIH, GI-2 and GI-3 show MIC $0.015 \mu \mathrm{g} / \mathrm{mL}$ against Klebsiella peneumoniae. It is worth noting that compound GI-1 displays the most prominent activity against all of the tested stains (MIC: 0.015-16 $\mu \mathrm{g} / \mathrm{mL}$ ).

\begin{tabular}{ccccccc}
\multicolumn{7}{c}{ TABLE } \\
\multicolumn{7}{c}{ in vitro ANTIBACTERIAL ACTIVITY OF } \\
GT-H AND ITS DERIVATIVES \\
Strains & \multicolumn{5}{c}{ MIC $(\mu \mathrm{g} / \mathrm{mL})$} \\
\cline { 2 - 7 } GI-H & S. e. & E. coli & P. a. & K. p. & A. b. & S. a. \\
\hline GI-1 & 0.015 & 1 & 32 & 0.015 & 1 & 1 \\
GI-2 & 32 & 64 & 256 & 0.015 & 16 & 16 \\
GI-3 & 6 & 2 & 8 & 0.015 & 0.5 & 0.5 \\
GI-4 & 128 & 256 & 256 & 256 & 256 & 256 \\
GI-5 & 32 & 128 & 256 & 256 & 256 & 256 \\
GI-6 & 256 & 128 & 256 & 256 & 256 & 256 \\
GI-7 & 256 & 256 & 256 & 256 & 256 & 256 \\
GI-8 & 256 & 256 & 256 & 256 & 256 & 256 \\
GI1-9 & 256 & 256 & 256 & 256 & 256 & 256 \\
GI-10 & 256 & 256 & 256 & 256 & 256 & 256 \\
GI-11 & 0.015 & 0.5 & 256 & 256 & 256 & 256 \\
GI-12 & 4 & 16 & 256 & 256 & 256 & 256 \\
\hline S.e. $=$ Staphylococcus epidermidis; P.a. = Pseudomonas aeruginosa; \\
K.p. = Klebsiella peneumoniae $;$ A.b. = Acinetobacter baumannii; \\
S.a. = Staphylococcus aureus R866.
\end{tabular}


In order to further know an effect of varying the N-4' substitution on activity, a series of novel synthesized quinolones were investigated. An interest in an influence of $\mathrm{N}$-substituent GI-H derivatives and unsubstituents, thus N-substituted compound GI-1 and GI-3 seems to have more inhibitory activity than unsubstituents GI-H, while better than other unsubstituents against both Gram-positive and Gram-negative bacteria. When compared to bulkier N-acyl compound GI-2, simple $\mathrm{N}$-acyl compound 7a display 16 to 2000 -fold increase in activity against all of the tested bacteria. Furthermore, this same trend can be seen in the comparison of N-sulfonyl substituted compounds GI-3 and GI-4.

\section{Conclusion}

In summary, gatifluoroxacin isomers GI-H, [1-cyclopropyl6-fluoro-1, 4-dihydro-8-methoxy-7-(2-methyl-1-piperazinyl)4-oxo-3-quinolinecarboxylic acid] and a series of its derivatives were designed and synthesized and evaluated for their in vitro antibacterial activity against representative Gram-positive and Gram-negative strains. Compounds GI1, GI2, GI3 and GI4 demonstrated excellent activity against both Gram-positive and Gram-negative bacteria. Compound GI-1 has been found to exhibit the most prominent activity against all of the tested stains (MIC: $0.015-16 \mu \mathrm{g} / \mathrm{mL}$ ).

As to SAR study among the thirteen novel synthesized quinolones, it have been demonstrated that the activity fluctuates in a broad range, depending on the substituents on the nitrogen atom of the $\mathrm{C}-7$ piperazine ring.

\section{ACKNOWLEDGEMENTS}

Financial support is provided by Sichuan Kelun Pharmaceutical Co. and Natural Science Foundation of China (J1103315/J0104).

\section{REFERENCES}

1. H. Koga, A. Itoh, S. Murayama, S. Suzue and T. Irikura, J. Med. Chem., 23, 1358 (1980).

2. (a) S. Emami, A. Shafiee and A. Foroumadi, Mini Rev. Med. Chem., 6, 375 (2006); (b) S.N. Pandeya, D. Sriram, G. Nath and E. De Clercq, Eur. J. Med. Chem., 35, 249 (2000); (c) A. Foroumadi, S. Emami, S. Pournourmohammadi, A. Kharazmi and A. Shafiee, Eur. J. Med. Chem. 40, 1346 (2005); (d) D.C. Hooper, Lancet Infect. Dis., 2, 530 (2002); (e) D.T. Bearden and L.H. Danziger, Pharmacotherapy, 21, 224S (2001); (f) L.J.V. Piddock, Drugs, 58(Supplement 2), 11 (1999).

3. (a) P.S. Lietman, Drugs, 49(Supplement 2), 159 (1995); (b) M.E. Falagas, P.I. Rafailidis and E.S. Rosmarakis, J. Antimicrob. Agents, 29, 374 (2007).

4. S.J. Projan, Curr. Opin. Pharmacol., 2, 513 (2002).

5. (a) K. Coleman, Drug Discov. Today, 1, 455 (2004); (b) A.V. Shindikar and C.L. Viswanathan, Bioorg. Med. Chem. Lett., 15, 1803 (2005).

6. (a) H. Kondo, F. Sakamoto, Y. Kodera and G. Tsukamoto, J. Med. Chem., 29, 2020 (1986); (b) H. Kondo, F. Sakamoto, T. Uno, Y. Kawahata and G. Tsukamoto, J. Med. Chem., 32, 671 (1989); (c) M.E. Jung, E.C. Yang, B.T. Vu, M. Kiankarimi, E. Spyrou and J. Kaunitz, J. Med. Chem., 42, 3899 (1999).

7. Z. Dang, Y.S. Yang, R.Y. Ji and S.H. Zhang, Bioorg. Med. Chem. Lett., 17, 4523 (2007).

8. R. Sánchez-Martín, J.M. Campos, A. Conejo-García, O. Cruz-López, M. Banez-Coronel, A. Rodríguez-González, M.A. Gallo, J.C. Lacal and A. Espinosa, J. Med. Chem., 48, 3354 (2005).

9. S.H. Zhao, R. Pine, J. Domagala and K. Drlica, Antimicrob. Agents Chemother., 43, 661 (1999).

10. (a) H. Schelleman, W.B. Bilker, C.M. Brensinger, F. Wan and S. Hennessy, Clin. Pharmacol. Ther., 88, 214 (2010); (b) F.J. Villasante, L. Gude, S.P. Fernandez, O. Alonso, E. Garcia and A. Cosme, Org. Process Res. Dev., 12, 900 (2008).

11. T.F. Ge, P.Y.P. Law, H.Y. Wong and Y.Y. Ho, Eur. J. Pharmacol., 573, 70 (2007).

12. Y. Mizuki, M. Kamaura, T. Yamaguchi, Y. Sekine and M. Hashimoto, Arzneimittelforschung, 39, 593 (1989).

13. T. Miyamoto, J. Matsumoto, K. Chiba, H. Egawa, K. Shibamori, A. Minamida, Y. Nishimura, H. Okada, M. Kataoka, M. Fujita, T. Hirose and J. Nakano, J. Med. Chem., 33, 1645 (1990).

14. A. Leonardi, G. Motta, C. Boi, R. Testa, E. Poggesi, P.G. De Benedetti and M.C. Menziani, J. Med. Chem., 42, 427(1999)

15. E. Ravina, C. Teran, L. Santana, N. Garcia and I. Estevez, Hetreocycles, 31, 1967 (1990).

16. D.A. Allemandi, F.L. Alovero and R.H. Manzo, J. Antimicrob. Chemother., 34, 261 (1994).

17. J.P. Sanchez, R.D. Gogliotti, J.M. Domagala, S.J. Gracheck, M.D. Huband, J.A. Sesnie, M.A. Cohen and M.A. Shapiro, J. Med. Chem., 38, 4478 (1995).

18. G. Anquetin, J. Greiner, N. Mahmoudi, M. Santillana-Hayat, R. Gozalbes, K. Farhati, F. Derouin, A. Aubry, E. Cambau and P. Vierling, Eur. J. Med. Chem., 41, 1478 (2006).

19. MICs were determined as described by the NCCLS (National Committee for Clinical Laboratory Standards. Performance Standards for Antimicrobial Susceptibility Testing: $11^{\text {th }}$ Informational Supplement; National Committee for Clinical Laboratory Standards: P.A. Wayne, 2001; Vol. 21, No. 1, M100-S11). The MIC was defined as the lowest concentration of each compound resulting in inhibition of visible growth of bacteria after incubation at $37{ }^{\circ} \mathrm{C}$ for $18-24 \mathrm{~h}$. 